The Rangeland Journal, 2014, 36, 259-265

http://dx.doi.org/10.1071/RJ13091

\title{
Foliar herbicide control of sticky florestina (Florestina tripteris DC.)
}

\author{
John McKenzie ${ }^{\mathrm{A}, \mathrm{B}}$, Dannielle Brazier ${ }^{\mathrm{A}}$, Shane Campbell ${ }^{\mathrm{A}, \mathrm{F}}$, Joseph Vitelli ${ }^{\mathrm{C}}$, \\ Angela Anderson ${ }^{\mathrm{D}}$ and Robert Mayer ${ }^{\mathrm{E}}$ \\ ABiosecurity Queensland, Department of Agriculture, Fisheries and Forestry, Tropical Weeds Research Centre, \\ PO Box 187, Charters Towers, Qld 4820, Australia. \\ B 241 Goomeribong Road, Goomeri, Qld 4601, Australia. \\ CBiosecurity Queensland, Department of Agriculture, Fisheries and Forestry, EcoSciences Precinct, PO Box 267, \\ Brisbane, Qld 4001, Australia. \\ DAgri-Science Queensland, Department of Agriculture, Fisheries and Forestry, Spyglass Beef Research Facility, \\ MS 99, Charters Towers, Qld 4820, Australia. \\ EMaroochy Research Station, 47 Mayers Road, Nambour, Qld 4560, Australia. \\ FCorresponding author. Email: Shane.Campbell@daff.qld.gov.au
}

\begin{abstract}
Sticky florestina (Florestina tripteris DC.) is an annual exotic weed that has become naturalised near the townships of Tambo and Barcaldine in central western Queensland, Australia. Three experiments conducted near Barcaldine identified foliar herbicides effective in killing sticky florestina plants and in providing residual activity to reduce recruitment from the soil seed bank. An initial chemical screening experiment evaluated the efficacy of 28 herbicide treatments. The most promising herbicides were then further evaluated in two response-rate experiments. Overall, 2,4-D/picloram, aminopyralid/fluroxypyr, clopyralid, metsulfuron-methyl and triclopyr/picloram proved to be the most effective selective herbicides. Two of these, metsulfuron-methyl at $18 \mathrm{~g}$ active ingredient (a.i) ha ${ }^{-1}$ and $^{-1}$ 2,4-D + picloram at $900 \mathrm{~g}$ a.i. $\mathrm{ha}^{-1}+225 \mathrm{~g}$ a.i. $\mathrm{ha}^{-1}$ have now been included in a minor use permit (PER11920) with the Australian Pesticides and Veterinary Medicines Authority (APVMA) for the control of sticky florestina in pasture, stock route, roadside and non-crop situations using both spot and boom-spray applications (APVMA 2010). The permit also allows the use of 2,4-D amine for the control of seedlings only.
\end{abstract}

Additional keywords: metsulfuron-methyl, picloram, rangelands, residual, triclopyr.

Received 4 September 2013, accepted 7 April 2014, published online 22 May 2014

\section{Introduction}

Florestina tripteris DC. (sticky florestina), family Asteraceae, is an annual exotic weed native to southern Texas, USA, and adjacent areas in Mexico (Turner 1963). It is believed to have been accidentally introduced into Australia in 1964 as a contaminant of buffel grass (Cenchrus ciliaris L.) seed from southern Texas and has since become naturalised near Tambo and Barcaldine in central western Queensland (Sparkes and Rogers 2007).

Sticky florestina has grey-green leaves, small white flowers and generally grows to a height of $15-60 \mathrm{~cm}$ (Turner 1963) although Sparkes and Rogers (2007) reported plants up to $1 \mathrm{~m}$ in height near Barcaldine. Its growth habit and flower structure are similar to those of parthenium (Parthenium hysterophorus L.), a major herbaceous weed in central Queensland, which was also accidentally introduced in pasture seed from the USA (Navie et al. 1996). Sticky florestina produces hundreds of sticky seeds, which are easily spread by stock, machinery and other vehicles (Sparkes and Rogers 2007). Seeds germinate quickly after rain and sticky florestina can complete a life cycle within a month if there is sufficient soil moisture (Sparkes and Rogers 2007). Anecdotally, sticky florestina may also act as a biennial, growing from the crown if conditions permit. In its native range, sticky florestina is found mainly along roadsides and in disturbed sites on various soils, from near sea level to $\sim 900 \mathrm{~m}$ (Turner 1963). Sticky florestina may cause cyanide poisoning in stock (Boughton and Hardy 1939; Blood et al. 2006).

As there were no registered herbicides for sticky florestina control in Australia, Sparkes and Rogers (2007) conducted a herbicide-screening experiment that resulted in an Australian Pesticides and Veterinary Medicines Authority (APVMA) restricted permit (PER7660 then renewed to PER9629 - now expired) for the control of sticky florestina using spot, highvolume or restricted-boom applications. This paper reports a further chemical screening experiment and two response-rate experiments, the last of which was undertaken using a boom spray 
to progress more broad-scale applications for treatment of extensive sticky florestina infestations.

\section{Materials and methods}

\section{Site details}

The experimental sites were on a cattle property $\left(23^{\circ} 48^{\prime} \mathrm{S}\right.$, $\left.145^{\circ} 12^{\prime} \mathrm{E}\right) \sim 50 \mathrm{~km}$ from Barcaldine. The landform was flat to gently sloping undulating plains with gilgai (small depressions) development throughout. The soils were primarily shrinking and expanding deep red, brown and grey cracking clays with scattered surface gravel or light stone cover. After historic clearing, the vegetation at the site was predominantly regenerating gidyea (Acacia cambagei R.T. Baker) and boree (Acacia tephrina Pedley) with the ground cover largely dominated by buffel grass (C. ciliaris L.), annual flinders grass (Iseilema vaginiflorum Domin), sticky florestina and several seasonal forbs. Rainfall reported is estimated from SILO data drill output (Natural Resources and Water 2009).

\section{Chemical screening experiment (Experiment 1)}

A randomised complete block experimental design was used with 28 treatments replicated four times. Though conditions were dry, sticky florestina was found growing in gilgais where soil moisture was higher. Plots $(2 \mathrm{~m} \times 2 \mathrm{~m})$ were centred over gilgais and only plots containing $>20$ well established sticky florestina plants were used.

Sixteen herbicides, some at two or three rates, were selected for screening based on registrations for control of other dicotyledonous (broad-leaf) weeds such as parthenium. Additionally, the herbicide mixture from the expired minor use permit (PER9629) and a control were included (Table 1). All herbicide treatments included a spray adjuvant (Table 1) and were applied in mid September 2007 using an Ag-murf pressurised sprayer at a spray volume of $1500 \mathrm{~L} \mathrm{ha}^{-1}$. Control plots were also sprayed with equivalent amounts of water and adjuvant.

The number of live sticky florestina plants present was counted in all plots 21 (8 October 2007), 67 (23 November 2007) and 164 (28 February 2008) days after treatment (DAT), with 1, 138 and $298 \mathrm{~mm}$ of rain falling between visits (estimated by Silo data drill output), including the initial herbicide application. The first two assessments quantified the efficacy of herbicides on original plants, while the last provided an indication of the herbicides' ability to minimise seedling recruitment, with abundant soil moisture available for germination and seedling emergence. Selective broad-leaf herbicide treatments that demonstrated low population counts $\left(\leq 5\right.$ plants $\left.\mathrm{m}^{-2}\right)$ at 164 DAT were monitored twice more, 205 (9 April 2008) and 276 (19 June 2008) DAT.

\section{First response-rate experiment (Experiment 2)}

The first response-rate experiment, initiated in late February 2008, was a randomised complete block design with 22 treatments replicated four times (Table 2). Plot size and layout were the same as in the screening experiment, with each plot containing at least 20 healthy adult sticky florestina plants. An adult plant was one that had commenced flowering.
The herbicides, 2,4-D amine, 2,4-D/picloram, aminopyralid/ fluroxypyr, clopyralid, metsulfuron-methyl, picloram and triclopyr/picloram, were each tested at three rates. The chemicals, 2,4-D amine and picloram, were included to try to determine which active ingredient was having the more potent effect in the formulations containing 2,4-D/picloram and triclopyr/picloram. A control treatment was included with plots only sprayed with equivalent amounts of water and adjuvant to those used in the herbicide treatments.

Herbicides were applied as in the screening experiment, except for picloram granules, which were mixed with sand to ensure a more even coverage when distributed over the plot. All plants were actively growing at the time of application.

Plant counts of sticky florestina were conducted at 42 (9 April 2008), 114 (20 June 2008), 132 (8 July 2008), 197 (11 September 2008) and 239 (23 October 2008) DAT, with 3, 32, 0, 82 and $55 \mathrm{~mm}$ of rain falling between visits (estimated by SILO data drill output), including the initial herbicide application.

\section{Second response-rate experiment (Experiment 3)}

The second response-rate experiment (which commenced in mid March 2009) was a randomised complete block design and was replicated four times, using larger plot sizes than in the previous experiments. Two rates each of 2,4-D/picloram, metsulfuronmethyl and triclopyr/picloram, along with a control treatment (sprayed with water and adjuvant only), were tested to refine herbicide rates (Table 3 ). Each $50 \times 4$-m plot had two randomly located $2 \times 2$-m permanent quadrats, in which all living sticky florestina plants were counted, and the results averaged. Plants were sprayed at a rate of $67 \mathrm{~L} \mathrm{ha}^{-1}$ using a 4-m boom spray attached to an ATV travelling at $10 \mathrm{~km} \mathrm{~h}^{-1}$. Agrotop TC110/02 nozzles were used and the pressure was $207 \mathrm{kPa}$. Adult (flowering) plants per quadrat were counted at 57 DAT (7 May 2009) and adult and juvenile plants (not flowering) at 96 (15 June 2009) DAT, with 52 and $21 \mathrm{~mm}$ of rain falling between visits (estimated by SILO data drill output), including the initial herbicide application. Unfortunately, due to excessive rainfall (>780 $\mathrm{mm}$ ), the experiment then had to be abandoned.

\section{Statistical analysis}

Density data for all three experiments were transformed using $\sqrt{ }(X+0.5)$, where $X=$ density, before statistical analysis and means were then back-transformed for presentation in tabular format in Tables 1-3. GENSTAT (2008) (Version 11.1.0.1575) was used for all statistical analyses, which involved ANOVA to identify significant treatment effects and Fisher's protected least significant difference test to identify differences among individual treatments. Following each analysis of transformed data, the residuals were tested for Normality and also for equality of variance to confirm that the square-root transformation was appropriate.

\section{Results}

\section{Chemical screening experiment}

Live sticky florestina plant numbers in each herbicide treatment were compared with control plant numbers (Table 1). The maximum reduction of initial sticky florestina plants was recorded at 67 DAT (23 November 2007), with significantly 
Table 1. Herbicides, rates and associated changes in plant density 21, 67, 164 and 205 days after treatment (DAT) during the 2007 screening experiment (Experiment 1) for the control of sticky florestina (Florestina triperis DC.) near Barcaldine, Queensland

Within columns values followed by the same letter are not significantly different $(P>0.05)$

\begin{tabular}{|c|c|c|c|c|c|c|}
\hline \multirow[t]{2}{*}{ Active ingredient ${ }^{\mathrm{A}}$} & \multirow[t]{2}{*}{ Trade name } & \multirow[t]{2}{*}{ Rate $\left(\mathrm{g}\right.$ a.i. $\mathrm{ha}^{-1}$ ) } & \multicolumn{4}{|c|}{ Plants $\mathrm{m}^{-2}$} \\
\hline & & & $21 \mathrm{DAT}$ & 67 DAT & 164 DAT & 205 DAT \\
\hline 2,4-D amine $\left(625 \mathrm{~g} \mathrm{~L}^{-1}\right)$ & Amicide 625 & 1250 & $35 \mathrm{abc}$ & 8abcdef & $8 \mathrm{abcd}$ & - \\
\hline 2,4-D amine $\left(625 \mathrm{~g} \mathrm{~L}^{-1}\right)$ & Amicide 625 & 2500 & $48 \mathrm{cde}$ & 7abcdef & $7 \mathrm{abcd}$ & - \\
\hline $\begin{array}{l}\text { 2,4-D amine } \\
\quad\left(625 \mathrm{~g} \mathrm{~L}^{-1}\right)+\text { metsulfuron- } \\
\quad \text { methyl }\left(600 \mathrm{~g} \mathrm{~kg}^{-1}\right)^{\mathrm{B}}\end{array}$ & Amicide 625/Brush-Off & $1875+180$ & $52 \mathrm{de}$ & $0 \mathrm{a}$ & $0 \mathrm{abc}$ & 0a \\
\hline $\begin{array}{l}\text { 2,4-D }\left(300 \mathrm{~g} \mathrm{~L}^{-1}\right) / \text { picloram } \\
\quad\left(75 \mathrm{~g} \mathrm{~L}^{-1}\right)\end{array}$ & Tordon 75-D & $450 / 112.5$ & $42 \mathrm{bcde}$ & $2 \mathrm{abcd}$ & $1 \mathrm{abc}$ & $0 \mathrm{a}$ \\
\hline $\begin{array}{l}\text { 2,4-D }\left(300 \mathrm{~g} \mathrm{~L}^{-1}\right) / \text { picloram } \\
\quad\left(75 \mathrm{~g} \mathrm{~L}^{-1}\right)\end{array}$ & Tordon 75-D & $900 / 225$ & 40abcd & $2 \mathrm{abcd}$ & $0 \mathrm{abc}$ & $1 \mathrm{a}$ \\
\hline $\begin{array}{l}\text { Aminopyralid }\left(10 \mathrm{~g} \mathrm{~L}^{-1}\right) / \\
\text { fluroxypyr }\left(140 \mathrm{~g} \mathrm{~L}^{-1}\right)\end{array}$ & Hotshot & $40 / 560$ & $45 \mathrm{bcde}$ & $3 a b c d$ & $3 a b c d$ & $4 a$ \\
\hline Chlorsulfuron $\left(750 \mathrm{~g} \mathrm{~kg}^{-1}\right)$ & Glean & 15 & 46bcde & 7abcdef & 17bcde & - \\
\hline Clopyralid $\left(300 \mathrm{~g} \mathrm{~L}^{-1}\right)$ & Lontrel & 93.75 & $53 \mathrm{de}$ & 2abcd & 19bcde & - \\
\hline Clopyralid $\left(300 \mathrm{~g} \mathrm{~L}^{-1}\right)$ & Lontrel & 187.5 & $42 \mathrm{bcde}$ & 9bcdefg & 4abcd & $9 \mathrm{a}$ \\
\hline Dichlorprop $\left(600 \mathrm{~g} \mathrm{~L}^{-1}\right)$ & Lantana 600 & 1800 & 43bcde & 10cdefgh & $24 \mathrm{def}$ & - \\
\hline Dichlorprop (600 g L-1) & Lantana 600 & 3600 & $48 \mathrm{cde}$ & $10 \mathrm{bcdefg}$ & 20cde & - \\
\hline DSMA $\left(220 \mathrm{~g} \mathrm{~L}^{-1}\right)$ & DSMA Clear & 750 & 4labcde & 8abcdef & 24def & - \\
\hline Fluroxypyr $\left(200 \mathrm{~g} \mathrm{~L}^{-1}\right)$ & Starane 200 & 150 & $50 \mathrm{cde}$ & $28 \mathrm{gh}$ & 130hij & - \\
\hline Fluroxypyr $\left(200 \mathrm{~g} \mathrm{~L}^{-1}\right)$ & Starane 200 & 300 & $42 \mathrm{bcde}$ & 11cdefgh & $59 \mathrm{efg}$ & - \\
\hline $\begin{array}{l}\text { Glufosinate-ammonium } \\
\qquad\left(200 \mathrm{~g} \mathrm{~L}^{-1}\right)\end{array}$ & Basta & 500 & $48 \mathrm{cde}$ & $26 \mathrm{gh}$ & $79 \mathrm{gh}$ & - \\
\hline $\begin{array}{l}\text { Glufosinate-ammonium } \\
\qquad\left(200 \mathrm{~g} \mathrm{~L}^{-1}\right)\end{array}$ & Basta & 1000 & $28 \mathrm{a}$ & 13defgh & 94ghi & - \\
\hline Glyphosate $\left(360 \mathrm{~g} \mathrm{~L}^{-1}\right)$ & Roundup & 540 & $36 a b c$ & $29 \mathrm{~h}$ & $189 \mathrm{j}$ & - \\
\hline Glyphosate $\left(360 \mathrm{~g} \mathrm{~L}^{-1}\right)$ & Roundup & 1080 & 49 cde & $16 \mathrm{efgh}$ & $78 \mathrm{gh}$ & - \\
\hline Imazapic $\left(240 \mathrm{~g} \mathrm{~L}^{-1}\right)$ & Flame & 90 & $52 \mathrm{de}$ & 10cdefgh & $59 \mathrm{efg}$ & - \\
\hline Imazapyr $\left(250 \mathrm{~g} \mathrm{~L}^{-1}\right)$ & Arsenal 250 & 750 & 43bcde & $2 \mathrm{abc}$ & 2abcd & - \\
\hline Metsulfuron-methyl $\left(600 \mathrm{~g} \mathrm{~kg}^{-1}\right)$ & Brush-Off & 4.2 & 46bcde & 4abcdef & $16 \mathrm{bcde}$ & - \\
\hline Metsulfuron-methyl $\left(600 \mathrm{~g} \mathrm{~kg}^{-1}\right)$ & Brush-Off & 8.4 & $56 \mathrm{e}$ & $1 \mathrm{ab}$ & $0 \mathrm{ab}$ & $1 \mathrm{a}$ \\
\hline Metsulfuron-methyl $\left(600 \mathrm{~g} \mathrm{~kg}^{-1}\right)$ & Brush-Off & 16.8 & $53 \mathrm{de}$ & $1 \mathrm{abc}$ & $0 \mathrm{ab}$ & $4 \mathrm{a}$ \\
\hline MSMA $\left(800 \mathrm{~g} \mathrm{~L}^{-1}\right)$ & Daconate & 8000 & $32 \mathrm{ab}$ & 10cdefgh & $162 \mathrm{ij}$ & - \\
\hline Pine oil $\left(680 \mathrm{~g} \mathrm{~L}^{-1}\right)$ & Organic interceptor & 204000 & 39abcd & $17 \mathrm{fgh}$ & 116ghij & - \\
\hline $\begin{array}{l}\text { Triclopyr }\left(300 \mathrm{~g} \mathrm{~L}^{-1}\right) / \text { picloram } \\
\quad\left(100 \mathrm{~g} \mathrm{~L}^{-1}\right)\end{array}$ & Grazon DS & $450 / 150$ & $52 \mathrm{de}$ & 4abcdef & 2abcd & $2 \mathrm{a}$ \\
\hline $\begin{array}{l}\text { Triclopyr }\left(300 \mathrm{~g} \mathrm{~L}^{-1}\right) / \text { picloram } \\
\qquad\left(100 \mathrm{~g} \mathrm{~L}^{-1}\right)\end{array}$ & Grazon DS & $900 / 300$ & $38 \mathrm{abcd}$ & $3 a b c d$ & $0 \mathrm{a}$ & $0 \mathrm{a}$ \\
\hline Control & - & - & $48 \mathrm{cde}$ & $15 \mathrm{efgh}$ & $73 \mathrm{fgh}$ & $143 \mathrm{~b}$ \\
\hline
\end{tabular}

${ }^{\mathrm{A}}$ All treatments included a spray adjuvant (Uptake) of paraffinic oil + alcohol alkloxylate $\left(582+240 \mathrm{~g} \mathrm{~L}^{-1}\right)$ at $4.37+1.8 \mathrm{~kg} \mathrm{a}^{\mathrm{i}}$. ha ${ }^{-1}$.

${ }^{\mathrm{B}}$ Herbicide mixture recommended in the expired minor use permit (PER9629).

$(P<0.05)$ lower plant densities than the control observed in treatments of 2,4-D amine + metsulfuron-methyl, 2,4-D/ picloram, aminopyralid/fluroxypyr, clopyralid (93.75 $\mathrm{g}$ a.i. $\mathrm{ha}^{-1}$ rate only), imazapyr, metsulfuron-methyl (rates of $8.4 \mathrm{~g}$ a.i. $\mathrm{ha}^{-1}$ and above) and triclopyr/picloram (900/300 g a.i. ha ${ }^{-1}$ rate only).

These herbicide treatments continued to maintain sticky florestina populations at low levels at 164 DAT (28 February 2008), even though the site had received $437 \mathrm{~mm}$ of rainfall posttreatment application (Table 1). In contrast, seedling recruitment in control plots resulted in an average density of 73 plants $\mathrm{m}^{-2}$ by 164 DAT.

Further monitoring of herbicide treatments with low population counts $\left(\leq 5\right.$ plants $\left.\mathrm{m}^{-2}\right)$ and controls at 164 DAT (except imazapyr) showed that, even at 205 DAT (9 April 2008), the selected rates of 2,4-D amine + metsulfuron-methyl, 2,4-D/ picloram, aminopyralid/fluroxypyr, clopyralid, metsulfuron- methyl and triclopyr/picloram continued to provide residual control (Table 1). However, by 276 DAT (19 June 2008) the residual activity had ceased, with sticky florestina density averaging 70 plants $\mathrm{m}^{-2}$ and no significant differences $(P>0.05)$ recorded between treatments (including the control).

\section{First response-rate experiment}

Population counts for 19 of the 21 herbicide treatments were significantly less $(P<0.05)$ than those in the control by 114 DAT (20 June 2008) (Table 2). The exceptions were the middle rate of picloram, which was not significantly different $(P>0.05)$ to the control, and the highest rate of 2,4-D amine, which had two and a half times more sticky florestina plants than the control.

Similar trends continued over subsequent recordings, although picloram treatments significantly $(P<0.05)$ reduced 
Table 2. Herbicides, rates and associated changes in plant density 42, 114, 132, 197 and 239 days after treatment (DAT) during the first response-rate experiment (2008) (Experiment 2) for the control of sticky florestina (Florestina triperis DC.) near Barcaldine, Queensland Within columns values followed by the same letter are not significantly different $(P>0.05)$

\begin{tabular}{|c|c|c|c|c|c|c|c|c|}
\hline \multirow[t]{2}{*}{ Active ingredient ${ }^{\mathrm{A}}$} & \multirow[t]{2}{*}{ Trade name } & \multirow[t]{2}{*}{ Rate $\left(\mathrm{g}\right.$ a.i. $\mathrm{ha}^{-1}$ ) } & \multicolumn{5}{|c|}{ Plants $\mathrm{m}^{-2}$} & \multirow[t]{2}{*}{ Cost $(A \$) h a^{-1 ~ B ~}$} \\
\hline & & & 42 DAT & $114 \mathrm{DAT}$ & 132 DAT & 197 DAT & 239 DAT & \\
\hline 2,4-D amine $\left(625 \mathrm{~g} \mathrm{~L}^{-1}\right)$ & Amicide 625 & 225 & 24de & 19bcde & $18 \mathrm{def}$ & $24 \mathrm{cde}$ & $30 \mathrm{de}$ & 2.07 \\
\hline 2,4-D amine $\left(625 \mathrm{~g} \mathrm{~L}^{-1}\right)$ & Amicide 625 & 450 & $25 \mathrm{e}$ & $40 \mathrm{e}$ & 18def & $49 \mathrm{e}$ & $48 \mathrm{e}$ & 4.14 \\
\hline 2,4-D amine $\left(625 \mathrm{~g} \mathrm{~L}^{-1}\right)$ & Amicide 625 & 900 & $25 \mathrm{e}$ & $207 \mathrm{~g}$ & $145 \mathrm{~h}$ & $182 \mathrm{f}$ & $180 \mathrm{f}$ & 8.28 \\
\hline $\begin{array}{l}\text { 2,4-D }\left(300 \mathrm{~g} \mathrm{~L}^{-1}\right) / \text { picloram } \\
\quad\left(75 \mathrm{~g} \mathrm{~L}^{-1}\right)\end{array}$ & Tordon 75-D & $225 / 56.25$ & $22 \mathrm{bcde}$ & $12 \mathrm{abcd}$ & 9abcde & $3 a b c$ & $7 \mathrm{abcd}$ & 9.32 \\
\hline $\begin{array}{l}\text { 2,4-D }\left(300 \mathrm{~g} \mathrm{~L}^{-1}\right) / \text { picloram } \\
\quad(75 \mathrm{~g} / \mathrm{L})\end{array}$ & Tordon 75-D & $450 / 112.5$ & $25 \mathrm{e}$ & $15 \mathrm{bcde}$ & $8 \mathrm{abcd}$ & $1 \mathrm{a}$ & 9abcd & 18.65 \\
\hline $\begin{array}{l}\text { 2,4-D }\left(300 \mathrm{~g} \mathrm{~L}^{-1}\right) / \text { picloram } \\
\quad\left(75 \mathrm{~g} \mathrm{~L}^{-1}\right)\end{array}$ & Tordon 75-D & $900 / 225$ & $16 \mathrm{~b}$ & $8 \mathrm{abc}$ & $1 \mathrm{ab}$ & $0 \mathrm{a}$ & $0 \mathrm{a}$ & 37.29 \\
\hline $\begin{array}{l}\text { Aminopyralid }\left(10 \mathrm{~g} \mathrm{~L}^{-1}\right) / \\
\text { fluroxypyr }\left(140 \mathrm{~g} \mathrm{~L}^{-1}\right)\end{array}$ & Hotshot & $10 / 140$ & 24de & $7 \mathrm{abc}$ & 4abcd & $1 \mathrm{ab}$ & $8 \mathrm{abcd}$ & 22.28 \\
\hline $\begin{array}{l}\text { Aminopyralid }\left(10 \mathrm{~g} \mathrm{~L}^{-1}\right) / \\
\text { fluroxypyr }\left(140 \mathrm{~g} \mathrm{~L}^{-1}\right)\end{array}$ & Hotshot & $20 / 280$ & 20bcde & $9 \mathrm{abc}$ & $4 \mathrm{abcd}$ & $1 \mathrm{ab}$ & $7 \mathrm{abcd}$ & 44.55 \\
\hline $\begin{array}{l}\text { Aminopyralid }\left(10 \mathrm{~g} \mathrm{~L}^{-1}\right) / \\
\text { fluroxypyr }\left(140 \mathrm{~g} \mathrm{~L}^{-1}\right)\end{array}$ & Hotshot & $40 / 560$ & 24de & $12 \mathrm{abcd}$ & 9abcde & $8 \mathrm{abcd}$ & 10abcd & 89.10 \\
\hline Clopyralid $\left(300 \mathrm{~g} \mathrm{~L}^{-1}\right)$ & Lontrel & 180 & $21 \mathrm{bcde}$ & 20bcde & $12 \mathrm{cde}$ & 9abcd & $23 \mathrm{cde}$ & $14.49^{\mathrm{C}}$ \\
\hline Clopyralid $\left(300 \mathrm{~g} \mathrm{~L}^{-1}\right)$ & Lontrel & 270 & $23 \mathrm{cde}$ & $15 \mathrm{bcde}$ & 10abcde & $3 a b c$ & $3 a b c$ & $21.73^{\mathrm{C}}$ \\
\hline Clopyralid $\left(300 \mathrm{~g} \mathrm{~L}^{-1}\right)$ & Lontrel & 360 & 20bcde & 10abcd & 10bcdef & $2 \mathrm{abc}$ & $1 \mathrm{ab}$ & $28.97^{\mathrm{C}}$ \\
\hline Metsulfuron-methyl $\left(600 \mathrm{~g} \mathrm{~kg}^{-1}\right)$ & Brush-Off & 4.2 & $26 \mathrm{e}$ & $26 \mathrm{cde}$ & 10bcde & 14abcde & 20bcde & 1.16 \\
\hline Metsulfuron-methyl $\left(600 \mathrm{~g} \mathrm{~kg}^{-1}\right)$ & Brush-Off & 8.4 & $17 \mathrm{bc}$ & 9abcd & 4abcd & $7 \mathrm{abcd}$ & 8abcd & 2.31 \\
\hline Metsulfuron-methyl $\left(600 \mathrm{~g} \mathrm{~kg}^{-1}\right)$ & Brush-Off & 36 & $10 \mathrm{a}$ & 1a & $0 \mathrm{a}$ & $0 \mathrm{a}$ & $0 \mathrm{a}$ & 9.90 \\
\hline Picloram $\left(20 \mathrm{~g} \mathrm{~kg}^{-1}\right)$ & Tordon Granules & 75 & $18 \mathrm{bcd}$ & $34 \mathrm{de}$ & $34 \mathrm{f}$ & 20bcde & $8 \mathrm{abcd}$ & 72.60 \\
\hline Picloram $\left(20 \mathrm{~g} \mathrm{~kg}^{-1}\right)$ & Tordon Granules & 150 & $26 \mathrm{e}$ & $42 \mathrm{ef}$ & $28 \mathrm{ef}$ & $32 \mathrm{de}$ & $31 \mathrm{de}$ & 145.20 \\
\hline Picloram $\left(20 \mathrm{~g} \mathrm{~kg}^{-1}\right)$ & Tordon Granules & 300 & $24 \mathrm{de}$ & $14 \mathrm{bcde}$ & $14 \mathrm{cdef}$ & $7 \mathrm{abcd}$ & 10abcd & 290.40 \\
\hline $\begin{array}{l}\text { Triclopyr }\left(300 \mathrm{~g} \mathrm{~L}^{-1}\right) / \text { picloram } \\
\quad\left(100 \mathrm{~g} \mathrm{~L}^{-1}\right)\end{array}$ & Grazon DS & $225 / 75$ & $22 \mathrm{cde}$ & $10 \mathrm{abcd}$ & 6abcd & $1 \mathrm{ab}$ & $5 \mathrm{abcd}$ & $15.82^{\mathrm{D}}$ \\
\hline $\begin{array}{l}\text { Triclopyr }\left(300 \mathrm{~g} \mathrm{~L}^{-1}\right) / \text { picloram } \\
\quad\left(100 \mathrm{~g} \mathrm{~L}^{-1}\right)\end{array}$ & Grazon DS & $450 / 150$ & $21 \mathrm{bcde}$ & $10 \mathrm{abcd}$ & $7 \mathrm{abcd}$ & $0 \mathrm{a}$ & 0a & $31.64^{\mathrm{D}}$ \\
\hline $\begin{array}{l}\text { Triclopyr }\left(300 \mathrm{~g} \mathrm{~L}^{-1}\right) / \text { picloram } \\
\quad\left(100 \mathrm{~g} \mathrm{~L}^{-1}\right)\end{array}$ & Grazon DS & $900 / 300$ & 21bcde & $4 a b$ & $3 a b c$ & $0 \mathrm{a}$ & $0 \mathrm{a}$ & $63.28^{\mathrm{D}}$ \\
\hline Control & - & - & $25 \mathrm{de}$ & $83 \mathrm{f}$ & $78 \mathrm{~g}$ & $141 \mathrm{f}$ & $152 \mathrm{f}$ & - \\
\hline
\end{tabular}

${ }^{\mathrm{A}}$ All treatments include a spray adjuvant (Uptake) of paraffinic oil + alcohol alkloxylate $\left(582+240 \mathrm{~g} \mathrm{~L}^{-1}\right)$ at $4.37+1.8 \mathrm{~kg}^{\mathrm{a} . i .}$ ha ${ }^{-1}$, which cost $\$ 53.63 \mathrm{ha}^{-1}$ based on prices as at December 2013.

${ }^{\mathrm{B}}$ Herbicide costs as at December 2013; labour not included.

${ }^{\mathrm{C}}$ Lontrel is no longer available as a $300 \mathrm{~g}^{-1}$ formulation so prices were calculated based on another product (Archer), which contained similar quantities of active ingredient.

${ }^{\mathrm{D}}$ Grazon DS is no longer produced so prices were calculated based on another product (Titan Picloram + Triclopyr), which contained similar quantities of active ingredient.

sticky florestina density at all rates thereafter. Plots treated with 2,4-D amine at the highest rate continued to have significantly higher $(P<0.05)$ plant densities than the control at 132 DAT $(8$ July 2008), but at 197 (11 September 2008) and 239 DAT (23 October 2008) they were not significantly different $(P>0.05)$ from each other. Many treatments showed declining counts after Day 114 even though their initial mortality rate was not high (e.g. clopyralid and 2,4-D/picloram).

At 239 DAT (23 October 2008), the lowest densities of sticky florestina $\left(\leq 10\right.$ plants $\left./ \mathrm{m}^{2}\right)$ were recorded in plots treated by all three rates of 2,4-D/picloram, aminopyralid/fluroxypyr, and triclopyr/picloram. This was also achieved by clopyralid and metsulfuron-methyl (at the two higher rates), and picloram at two application rates (Table 2). No sticky florestina plants were recorded 239 DAT at the highest rates of 2,4-D/picloram, metsulfuron-methyl, and triclopyr/picloram, nor at the medium rate of triclopyr/picloram. The cost of the herbicide mixture (excluding adjuvant) to apply these treatments was A $\$ 37.29$, A\$9.90, A\$63.28 and A\$31.64 ha ${ }^{-1}$, respectively.

\section{Second response-rate experiment}

The herbicides, 2,4-D/picloram, metsulfuron-methyl and triclopyr/picloram, all caused major reductions in the population density of sticky florestina at 57 DAT (7 May 2009) and at 96 DAT (7 May 2009) (Table 3). Most treated plants died within 2 months and there was no seedling recruitment at 96 DAT. At 96 DAT, the adult population in all herbicide treatments was significantly lower $(P<0.05)$ than the control and no treated plots had any juvenile sticky florestina plants although recruitment was also very low in the control plots (Table 3 ) averaging only $1 \mathrm{~m}^{-2}$. At the rates of herbicide applied, metsulfuron-methyl was by far the cheapest mixture (between $\mathrm{A} \$ 3.30$ and $\mathrm{A} \$ 4.95 \mathrm{ha}^{-1}$ ) followed by 2,4-D/picloram (between A $\$ 27.97$ and A 
Table 3. Herbicides, rates and associated changes in plant density 57 and 96 days after treatment (DAT) during the second response-rate experiment (2009) (Experiment 3) for the control of sticky florestina (Florestina triperis DC.) near Barcaldine, Queensland Within columns values followed by the same letter are not significantly different $(P>0.05)$

\begin{tabular}{|c|c|c|c|c|c|}
\hline Active ingredient $\mathrm{t}^{\mathrm{A}}$ & Trade name & $\begin{array}{c}\text { Rate } \\
\left(\mathrm{g} \mathrm{a} \text { a.i. } \mathrm{ha}^{-1}\right)\end{array}$ & $\begin{array}{c}\text { Adults } \\
\mathrm{m}^{-2} 57 \text { DAT }\end{array}$ & $\begin{array}{c}\text { Adults } \\
\mathrm{m}^{-2} 96 \text { DAT }\end{array}$ & $\begin{array}{l}\text { Cost } \\
(\mathrm{A} \$)^{-1 \mathrm{~B}}\end{array}$ \\
\hline 2,4-D $\left(300 \mathrm{~g} \mathrm{~L}^{-1}\right) /$ picloram $\left(75 \mathrm{~g} \mathrm{~L}^{-1}\right)$ & Tordon 75-D & $675 / 168.75$ & $1 a b c$ & $1 \mathrm{ab}$ & 27.97 \\
\hline 2,4-D $\left(300 \mathrm{~g} \mathrm{~L}^{-1}\right) /$ picloram $\left(75 \mathrm{~g} \mathrm{~L}^{-1}\right)$ & Tordon 75-D & $900 / 225$ & $0 \mathrm{a}$ & $0 \mathrm{a}$ & 37.29 \\
\hline Metsulfuron-methyl $\left(600 \mathrm{~g} \mathrm{~kg}^{-1}\right)$ & Brush-Off & 12 & $3 b c$ & $1 \mathrm{ab}$ & 3.30 \\
\hline Metsulfuron-methyl $\left(600 \mathrm{~g} \mathrm{~kg}^{-1}\right)$ & Brush-Off & 18 & $1 \mathrm{ab}$ & $0 \mathrm{a}$ & 4.95 \\
\hline Triclopyr $\left(300 \mathrm{~g} \mathrm{~L}^{-1}\right) /$ picloram $\left(100 \mathrm{~g} \mathrm{~L}^{-1}\right)$ & Grazon DS & $510 / 170$ & $5 \mathrm{c}$ & $2 b$ & $35.86^{\mathrm{C}}$ \\
\hline Triclopyr $\left(300 \mathrm{~g} \mathrm{~L}^{-1}\right) /$ picloram $\left(100 \mathrm{~g} \mathrm{~L}^{-1}\right)$ & Grazon DS & $690 / 230$ & $1 \mathrm{abc}$ & $1 \mathrm{ab}$ & $48.51^{\mathrm{C}}$ \\
\hline Control & - & - & $18 \mathrm{~d}$ & $18 \mathrm{c}$ & - \\
\hline
\end{tabular}

${ }^{\mathrm{A}}$ All treatments include a spray adjuvant (Uptake) of paraffinic oil + alcohol alkloxylate $\left(582+240 \mathrm{~g} \mathrm{~L}^{-1}\right)$ at $1.164+0.48 \mathrm{~kg}$ a.i. ha ${ }^{-1}$, which cost $\mathrm{A} \$ 14.30$ based on prices as at December 2013.

${ }^{\mathrm{B}}$ Herbicide costs as at December 2013; labour not included.

${ }^{\mathrm{C}}$ Grazon DS is no longer produced so prices were calculated based on another product (Titan Picloram + Triclopyr), which contained similar quantities of active ingredient.

$\$ 37.29 \mathrm{ha}^{-1}$ ) and then triclopyr/picloram (between A $\$ 35.86$ and A $\$ 48.51 \mathrm{ha}^{-1}$ ) (Table 3). At the rate applied, the cost of the adjuvant was $\mathrm{A} \$ 14.30 \mathrm{ha}^{-1}$.

\section{Discussion}

\section{Effective herbicides}

Six foliar herbicides initially demonstrated high efficacy of control against sticky florestina, three of these at two different

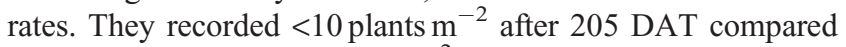
with an average of 143 plants $^{-2}$ in control plots (Table 1). Of these, 2,4-D/picloram, aminopyralid/fluroxypyr, clopyralid, metsulfuron-methyl and triclopyr/picloram were progressed to the response-rate experiments, with 2,4-D amine and picloram. Imazapyr, despite its significant effects earlier in the experiment, was excluded due to its non-selectivity and our observation that its efficacy was reduced in areas of high organic matter, such as dead-grass crowns and manure. In such areas, with absence of competition from grass and other herbaceous species, seedlings of sticky florestina quickly developed into a healthy monoculture.

The two mixtures, containing picloram as one of their active ingredients (2,4-D/picloram and triclopyr/picloram), consistently provided effective control of sticky florestina in the response-rate experiments (Tables 2 and 3). For 2,4-D/picloram, there was no marked difference in efficacy at the rates tested within the respective experiments using differing application methods. However, the application of 900/225 g a.i. $\mathrm{ha}^{-1}$, in all three experiments, resulted in the absence of sticky florestina plants at 96-239 DAT, depending on the duration of the particular experiment. In the final response-rate experiment, a rate of 675/ $168.75 \mathrm{~g}$ a.i. $\mathrm{ha}^{-1}$ was compared with $900 / 225 \mathrm{~g} \mathrm{ha}^{-1}$ to see if it offered a similar level of control but at a reduced cost. Due to unfavourable weather conditions, this experiment ended at 96 DAT, with both treatments exhibiting similar effects at that assessment.

Triclopyr/picloram was applied at rates of 225/75, 450/150, 510/170, 690/230 and 900/300 g a.i. ha ${ }^{-1}$ (Tables 1-3). The 510/ 170 and 690/230 rates were chosen in the boom-spray experiment to correspond with picloram levels in the 2,4-D/picloram rates of $675 / 168.75$ and $900 / 225 \mathrm{~g}$ a.i. ha ${ }^{-1}$. Mortality of sticky florestina plants was high for all rates of triclopyr/picloram; residual control was also high. Testing of 2,4-D amine and picloram on their own (Table 1) clearly showed that the prolonged control achieved with 2,4-D/picloram was associated with the picloram component. A similar result occurred with the use of aminopyralid/fluroxypyr (Table 1). Fluroxypyr on its own (Table 1) provided minimal control of sticky florestina, but the addition of aminopyralid resulted in good initial mortality rates and ongoing control. A new product, triclopyr/picloram/ aminopyralid (Dow AgroSciences 2013), has since come onto the market and, based on its active ingredients and our results, this formulation may be highly effective at controlling sticky florestina. 2,4-D amine when applied at recommended rates for some other weeds (Table 1) did provide high efficacy in the screening experiment. However, it is generally considered a knock-down herbicide and based on their findings, Sparkes and Rogers (2007) recommended it for initial control of sticky florestina but not for providing residual control of seedling regrowth. As such, in the present study, preference was given to 2,4-D/picloram, which included the more persistent picloram component.

For metsulfuron-methyl, seven rates were tested across the three experiments: $4.2,8.4,12,16.8,18,36$ and $180 \mathrm{~g}$ a.i. ha ${ }^{-1}$ (Tables 1-3), with the latter included in a mixture with 2,4-D amine (Table 1) based on the recommendations of Sparkes and Rogers (2007). All rates of metsulfuron-methyl eventually reduced sticky florestina populations. Rates equal to or above a relatively low minimum of $8.4 \mathrm{~g}$ a.i. $\mathrm{ha}^{-1}$ provided almost total control of populations for $2-6$ months (Tables 1-3). For example, no sticky florestina plants were recorded 164 days after spraying metsulfuron-methyl at a rate of $8.4 \mathrm{~g}$ a.i. ha ${ }^{-1}$, while untreated plots had 73 plants $\mathrm{m}^{-2}$ (Table 1). Sparkes and Rogers (2007) also reported prolonged control of sticky florestina using metsulfuron-methyl at $0.24 \mathrm{~g}$ a.i. $\mathrm{L}^{-1}$. In their study, seedling recruitment in treated plots was only $30 \%$ of that in untreated controls $\sim 12$ months after application.

Metsulfuron-methyl is registered in Australia for the control of the herbaceous weed, parthenium, in pastures at a rate of $4.2 \mathrm{~g}$ a.i. ha ${ }^{-1}$ (Dupont 2011). Recently it has also been found to kill plants of Pimelea spp. present in Queensland at rates as low 
as $3 \mathrm{~g}$ a.i. $\mathrm{ha}^{-1}$, but it could not be determined if any residual activity occurred at such a low rate (Silcock et al.2012). Based on replanting times of pasture species after the use of metsulfuronmethyl, the label suggests a timeframe of 8-12 weeks as an indication of how long the chemical may persist for at an application rate of $5 \mathrm{~g}$ a.i. $\mathrm{ha}^{-1}$. The duration increases to greater than 12 months for rates higher than $15 \mathrm{~g}$ a.i. $\mathrm{ha}^{-1}$. The label does emphasize, however, that the period could vary depending on site conditions such as climate, soil $\mathrm{pH}$, presence of soil microorganisms, soil temperature, soil moisture and the rate used (Dupont 2011). The work of Sparkes and Rogers (2007) led to an APVMA ( $\leq 1 \mathrm{ha})$ minor use permit (PER7660 then renewed to PER9629 - Expired) that allowed for the use of $200 \mathrm{~g} \mathrm{2,4-D}$ amine $+200 \mathrm{~mL}$ of biodegradable surfactant (BS1000) $100 \mathrm{~L}^{-1}$ or $100 \mathrm{~g} \mathrm{2,4-D}$ amine $+20 \mathrm{~g}$ metsulfuronmethyl $+200 \mathrm{~mL}$ BS1000 $100 \mathrm{~L}^{-1}$, depending on the life stage of the plant. The present study suggests that even lower rates of metsulfuron-methyl could be effectively applied, thereby reducing costs for broad-scale applications.

Clopyralid performed well, particularly at higher rates, which is consistent with the findings of Sparkes and Rogers (2007), who also found it to be effective when applied as a spotspray application at a rate of $6 \mathrm{~g}$ a.i. $\mathrm{L}^{-1}$.

\section{Non-target impacts}

All herbicides that progressed to the rate screening stages were selective broad-leaf chemicals, which generally resulted in no damage to the co-existing pasture grass species. Higher rates of metsulfuron-methyl ( 36 and $180 \mathrm{~g}$ a.i. ha ${ }^{-1}$ ) did, however, have some effect on grasses, particularly Flinders grass, which was the most sensitive species and disappeared from the plots at these rates. The metsulfuron-methyl labels mention that some grasses can be damaged if sprayed, although many species tend to recover (Dupont 2011). Popay et al. (1985), in one of the earlier studies on metsulfuron-methyl, found that rates of $18 \mathrm{~g}$ a.i. $\mathrm{ha}^{-1}$ damaged perennial ryegrass (Lolium perenne L.) and white clover (Trifolium repens L.) growing in New Zealand pastures.

Clovers and some other legumes are particularly sensitive to metsulfuron-methyl (Popay et al. 1985; Anderson and Panetta 1995; James et al. 1999), although the labels of all the effective herbicides identified in this study indicate that they will have deleterious impacts on some leguminous species (Dupont 2011; Dow AgroSciences 2013). For example, triclopyr/picloram at a rate of 300/100 g a.i. $\mathrm{L}^{-1}$ damaged white clover in Queensland pastures in a fireweed (Senecio madagascariensis Poir.) herbicide trial (Anderson and Panetta 1995). Similarly, 2,4-D/picloram at a rate of 780/200 g a.i. $\mathrm{ha}^{-1}$ and clopyralid at a rate of $300 \mathrm{~g}$ a.i. $\mathrm{ha}^{-1}$ completely removed white clover for the 3-month duration of a trial in New Zealand (James et al. 1999). While there is a paucity of information on potential non-target impacts where sticky florestina is growing in western Queensland, some native herbage (including legumes) is present and would most likely be damaged by the effective chemicals identified in this study. Some of the desirable species that could be affected include saltbushes (Atriplex spp.), ruby saltbush (Enchylaena tomentose R.BR), climbing saltbush [Einadia nutans (R.BR.) A.J.Scott], cow vine (Ipomea lonchophylla J.M.Black), fringed glycine (Glycine falcata Benth.), rhynchosia [Rhynchosia minima (L.) DC] and tar vines (Boerhavia spp.) (Milson 2002). However, opportunities for re-establishment from the seed bank or neighbouring plants should occur following control of sticky florestina, particularly after spot spraying.

\section{Treatment costs}

Metsulfuron-methyl was by far the cheapest herbicide to apply at an effective rate. In the second response-rate experiment, when applied at $18 \mathrm{~g}$ a.i. $\mathrm{ha}^{-1}$, the herbicide mixture (excluding adjuvant) cost $A \$ 4.95 \mathrm{ha}^{-1}$. In contrast, 2,4-D/picloram at 900/ $225 \mathrm{~g}$ a.i. $\mathrm{ha}^{-1}$, and triclopyr/picloram at 510/170 g a.i. ha ${ }^{-1}$, cost $\mathrm{A} \$ 37.29$ and $\mathrm{A} \$ 35.86 / \mathrm{ha}^{-1}$, respectively, for an equivalent degree of control. The cost of the adjuvant component varied considerably between experiments, depending on the rate applied. In Experiment $2\left(\mathrm{~A} \$ 53.63 \mathrm{ha}^{-1}\right)$, the application was based on applying $500 \mathrm{~mL}$ of product per $100 \mathrm{~L}$ of water, while in Experiment 3 it was based on recommended rates for boomspray applications ( $2 \mathrm{~L}$ of product $\mathrm{ha}^{-1}$ ) and was consequently much cheaper $\left(\mathrm{A} \$ 14.30 \mathrm{ha}^{-1}\right)$. It is feasible that even lower rates of adjuvant could be used and this warrants further investigation.

\section{Management considerations}

The most effective herbicides observed in this study demonstrated high efficacy under both low $\left(67 \mathrm{~L} \mathrm{ha}^{-1}\right.$, Table 3$)$ and high volume (1500 $\mathrm{L} \mathrm{ha}^{-1}$, Tables 1 and 2) applications. This is despite the experiments being implemented at different seasons (ranging from early spring to early autumn) and being exposed to different environmental conditions (e.g. temperatures, humidity and rainfall). This should provide land managers with some flexibility in the selection and timing of the most appropriate method of application. Options could range from spot-spraying small patches to broad-scale control of large infestations using boom-spray equipment. The latter would be restricted to areas of suitable terrain and may not be appropriate in pulled gidyea country, which is characteristically rough and with numerous depressions (gilgais). For these situations, aerial applications may be worth exploring in the future, particularly if infestations become too large to treat cost-effectively using ground-based techniques.

For broad-scale herbicide control of sticky florestina in pasture situations, metsulfuron-methyl would be the most costeffective option based on current herbicide prices. However, an international survey of herbicide resistant weeds has identified 62 reported cases of resistance to metsulfuron-methyl, involving 31 weed species (Heap 2014). To minimise this risk, it is recommended that land managers rotate the use of metsulfuronmethyl with other chemicals, particularly ones with a different mode of action (Vitelli and Pitt 2006). This study has identified several effective herbicides (e.g. 2,4-D/picloram, triclopyr/ picloram) with the disruptors of plant cell growth mode of action (Group I herbicides) that could be used in rotation with metsulfuron-methyl, which has the inhibitor of the enzyme acetolactate synthase mode of action (Group B herbicide). Although more expensive, herbicides containing active ingredients (such as picloram and aminopyralid) that are known to provide residual control may be good options for areas where dense sticky florestina populations occur. These herbicides all 
have the added advantage of being selective against broad-leaf weeds and, as such, do not damage any grasses that may be present. This is highly pertinent, as sticky florestina appears to be an opportunistic weed that prefers disturbed environments such as roadsides, around watering points and heavily grazed areas (Turner 1963). Maintaining a healthy pasture should therefore help prevent the establishment and spread of sticky florestina.

The timing of herbicide application will depend on rainfall, as in western Queensland sticky florestina appears capable of germinating at any time of year if there is sufficient soil moisture (Sparkes and Rogers 2007). In years when rainfall follows the normal seasonal patterns (distinct wet and dry seasons), spraying after the first significant rainfall event of the wet season (generally late spring/early summer) would be advantageous, provided it is done before new plants reach reproductive maturity. At this stage, effective herbicides should cause high mortality of any sticky florestina plants present and then provide residual control for all or a large portion of the summer. When seedlings start to appear again after rainfall events, land managers need to spray within $\sim 4$ weeks to prevent completion of the life cycle and replenishment of the soil seed bank.

If all existing sticky florestina plants can be controlled before becoming reproductive, and there is no re-infestation from neighbouring areas, the duration of a sticky florestina control program will be dependent on the longevity of the soil seed bank. Roads near the research site were regularly sprayed to reduce the risk of spread of weed seeds. The pattern of seedling recruitment in these areas tended to indicate that the sticky florestina seed bank may be relatively short-lived (perhaps a couple of years).

\section{Conclusion}

As a result of this research, effective herbicides and rates for the broad-scale control of sticky florestina have been identified. They are consistent with the earlier recommendations of Sparkes and Rogers (2007), but refinement of rates has identified lower quantities of active ingredient that can be applied for some herbicides (such as metsulfuron-methyl), particularly for broadscale applications, while maintaining efficacy.

The APVMA has now approved a minor use permit (PER11920) (APVMA 2010) allowing for two of the effective herbicides identified from this study (metsulfuron-methyl at $18 \mathrm{~g}$ a.i. $\mathrm{ha}^{-1}$ and 2,4-D/picloram at 900/225 g a.i. ha ${ }^{-1}$ ) to be applied in pastures, stock routes, roadsides and non-crop situations. A registration for the control of seedlings only using 2,4-D amine at $1200 \mathrm{~g}$ a.i. ha ${ }^{-1}$ was also included in the permit, based on the present study and the previous work of Sparkes and Rogers (2007). Additionally, sticky florestina has been added to the FallowBoss Tordon Herbicide label at 900/225/22.5 (2,4-D/ picloram/aminopyralid, respectively) g a.i. $\mathrm{ha}^{-1}$. The product is to be applied using a boomspray in agricultural non-crop areas, commercial and industrial areas, pastures and rights of way.

\section{Acknowledgements}

The authors acknowledge the financial support from Desert Channels Queensland. Also a special thanks to John and Jocelyn Chandler for providing the field experimental site. The manuscript was reviewed by Dr Dane Panetta, Dr Wayne Vogler and Barbara Madigan before submission and we are grateful for their constructive comments.

\section{References}

Anderson, T. M. D., and Panetta, F. D. (1995). Fireweed response to boomspray applications of different herbicides and adjuvants. Plant Protection Quarterly 10, 152-153.

APVMA (2010). Permit (PER11920) for control of Florestina tripteris in pastures and stock routes, roadside verges and non-crop situations. Available at: http://permits.avpma.gov.au/PER11920.PDF (accessed 16 July 2012).

Blood, D. C., Studdert, V. P., and Gay, C. C. (2006). 'Saunders Comprehensive Veterinary Dictionary.' 3rd edn. (Saunders Elsevier: St Louis, MO.)

Boughton, I. B., and Hardy, W. T. (1939). Hard yellow livers of sheep and cattle and feeding trials of suspected plants. Texas Agricultural Experimental Station Annual Report 52, 238-240.

Dow AgroSciences (2013). 'The Product Guide 2013.' (Dow AgroSciences: Frenchs Forest, NSW.)

Dupont (2011). Dupont crop protection product manual. Available at: www2. dupont.com/Crop_Protection/en_AU/products/DuPont\%20Crop\%20 Protection\%20Product\%20Manual\%20September\%202011.pdf (accessed 28 January 2014).

Genstat (2008). Available at: www.itradersdvd.com/3tbb1/loadfiles.php? $\mathrm{id}=412870$ (accessed 25 March 2014).

Heap, I. (2014). The international survey of herbicide-resistant weeds. Available at: www.weedscience.com (accessed 29 January 2014).

James, T. K., Rahman, A., and Cornwell, M. J. (1999). Pasture tolerance to the herbicide metsulfuron-methyl. In: 'Proceedings of the 52nd Plant Protection Conference'. New Zealand. (Ed. M. O'Callaghan.) pp. 240-244. (The New Zealand Plant Protection Society Inc.: Havelock North, New Zealand.)

Milson, J. (2002). 'Pasture Plants of North-west Queensland.' (Department of Natural Resources: Brisbane, Qld.)

Natural Resources and Water (2009). Enhanced Meteorological Datasets, SILO Data Drill. Available at: www.longpaddock.qld.gov.au/silo/ (accessed 19 April 2014).

Navie, S. C., McFadyen, R. E., Panetta, F. D., and Adkins, S. W. (1996). The biology of Australian weeds 27. Parthenium hysterophorus L. Plant Protection Quarterly 11, 76-88.

Popay, A. I., Cornwell, M. J., and Rahman, A. (1985). Effects of metsulfuronmethyl on pasture. In: 'Proceedings of the 38th New Zealand Weed and Pest Control Conference'. Hastings, New Zealand. (Ed. M. J. Hartley.) pp. 102-105. (The New Zealand Weed and Pest Control Society Inc.: Havelock North, New Zealand.)

Silcock, R. G., Mann, M. B., Chow, S., and Vitelli, J. S. (2012). Herbicides to control poisonous Pimelea species (Thymelaeaceae). Crop Protection 31, 99-106. doi:10.1016/j.cropro.2011.09.013

Sparkes, E. C., and Rogers, M. (2007). Sticky florestina (Florestina triperis DC.Prod.) herbicide screening for a recent invasive species into far western Queensland. In: 'Proceedings of the 21st Asian Pacific Weed Science Society Conference'. Colombo, Sri Lanka. (Eds B. Marambe, U. R. Sangakkara, W. A. J. M. De Costa and A. S. K. Abeysekara.) pp. 463-468. (Asian Pacific Weed Science Society: Colombo, Sri Lanka.)

Turner, B. L. (1963). Taxonomy of Florestina (Helenieae, Compositae). Brittonia 15, 27-46. doi:10.2307/2805037

Vitelli, J. S., and Pitt, J. L. (2006). Assessment of current weed control methods relevant to the management of the biodiversity of Australian rangelands. The Rangeland ,Journal 28, 37-46. doi:10.1071/RJ06016 